•研究报告・

\title{
不同放牧强度对赛罕乌拉草原蜘蛛多样性的影响
}

\author{
张宇@1\#，王露雨①\#，向昌林 ${ }^{2}$, 段美春( $3^{*}$, 张志升@1*
}

1. 西南大学生命科学学院三峡库区生态环境教育部重点实验室, 重庆 400715; 2. 赛罕乌拉国家级自然保护区管理局, 内蒙古赤峰 025150;

3. 西南大学农学与生物科技学院, 重庆 400715

摘要: 蜘蛛作为草原生态系统中的主要消费者, 对维系草原生物多样性和生态系统功能具有重大意义。放牧是人类利用草原 最普遍的方式, 了解放牧对蜘蛛多样性的影响具有重要生态学意义。本研究调查了内蒙古赛罕乌拉草原上 5 个不同放牧强度 样地中的蜘蛛多样性, 通过单因素方差分析(one-way analysis of variance)比较各样地中的蜘蛛多样性, 非度量多维标度分析 (non-metric multidimensional scale, NMDS)和相似性分析(analysis of similarities, ANOSIM) 比较各样地间的蜘蛛物种组成相似 性，再结合相关性分析探讨了植被高度对蜘蛛多样性的影响。结果表明：重度放牧强度样地的蜘蛛多样性显著低于其他未放 牧及轻度放牧样地; 具体到常见科上, 放牧强度对织网型的园蛛物种数和个体数影响显著, 而对游猎型的狼蛛、跳蛛却不明 显; 织网型蜘蛛主要受植被结构影响, 而游猎型蜘蛛更可能受潜在猎物可得性的影响。NMDS分析表明不同放牧强度下, 蜘 蛛类群的物种组成呈现明显的梯度变化, 放牧强度越低, 物种组成和未放牧样地越相近。相关性分析表明草原植被高度与蜘 蛛多样性总体上呈正相关关系, 即植被高度越高, 蜘蛛多样性越高。其中依靠植物构建蛛网的园蛛科和在植物上层伏击猎物 的蟹蛛科、逍遥蛛科等与植被高度显著相关。这说明植物资源及其空间异质性可能对草原蜘蛛多样性起着主导作用。因此, 降 低放牧强度有助于保护草原蜘蛛群落的多样性, 特别有利于织网型蜘蛛。

关键词: 草原生态系统; 放牧强度; 织网型蜘蛛; 游猎型蜘蛛; 功能群

张宇，王露雨，向昌林，段美春，张志升 (2021) 不同放牧强度对赛罕乌拉草原蜘蛛多样性的影响. 生物多样性，29，467-476. doi: 10.17520/ biods.2020214.

Zhang Y, Wang LY, Xiang CL, Duan MC, Zhang ZS (2021) Effects of different grazing intensities on spider diversity in Saihanwula Grassland. Biodiversity Science, 29, 467-476. doi: 10.17520/biods.2020214.

\section{Effects of different grazing intensities on spider diversity in Saihanwula Grassland}

Yu Zhang ${ }^{(1 \#}$, Luyu Wang ${ }^{(1 \#}$, Changlin Xiang ${ }^{2}$, Meichun Duan ${ }^{\left(13^{*}\right.}$, Zhisheng Zhang ${ }^{(1 *}$

1 Key Laboratory of Eco-Environments in Three Gorges Reservoir Region (Ministry of Education), School of Life Sciences, Southwest University, Chongqing 400715

2 Saihanwula National Nature Reserve Administration, Chifeng, Inner Mongolia 025150

3 College of Agronomy and Biotechnology, Southwest University, Chongqing 400715

\section{ABSTRACT}

Aims: As the main consumers of grassland ecosystem, spiders are of great significance to maintain biodiversity and ecosystem function in grasslands. Grazing is the most common way for humans to exploit grasslands, so it is ecologically important to understand the effects of grazing on the spider diversity. In this study, we selected five sample plots that varied in their grazing intensity in the Saihanwula Grassland in Inner Mongolia.

Methods: We analyzed differences in spider diversity and species composition among these five sample sites using one-way analysis of variance tests and non-metric multidimensional scale analysis (NMDS) and analysis of similarities (ANOSIM), respectively. We also evaluated the effect of vegetation height on spider biodiversity using a correlation analysis.

Results: Spider biodiversity in heavily grazed sites is significantly lower than in non-grazed and lightly grazed sites.

收稿日期: 2020-05-26; 接受日期: 2020-12-09

基金项目: 科技部基础调查专项(2018FY100305)、国家自然科学基金(31672278)和重庆市研究生科研创新项目(CYS20104)

\# 共同第一作者 Co-first authors

* 共同通讯作者 Co-authors for correspondence. E-mail: duanmc@swu.edu.cn; zhangzs327@qq.com

https://www.biodiversity-science.net 
Grazing intensity also had a significant effect on spider richness and the abundance of garden spiders, but not on the number of wolf and jumping spiders. Web-building spiders were mainly affected by vegetation structure, while hunting spiders were more likely to be affected by potential prey availability. NMDS analysis revealed that species composition significantly varied across sites of different grazing intensities. Sites with lower grazing intensities had more similar composition to non-grazed sites than sites that were heavily grazed. The correlation analysis showed that the height of grassland vegetation was positively related to spider biodiversity. Spiders from the family Araneidae (which build webs on plants), Thomisidae and Philodromidae (which ambush prey on the upper layer of plants) were highly correlated with vegetation height.

Conclusions: Our results suggest that available resources and spatial heterogeneity of habitat could play a leading role in supporting high grassland spider diversity. Therefore, reducing grazing intensity can contribute to the maintenance of grassland spider diversity community composition, especially for web-building spiders that depend on the vegetation structure provided by intact grasslands.

Key words: grassland ecosystem; grazing intensity; web-building spiders; hunting spiders; functional group; biodiversity

草地是我国陆地上面积最大的生态系统, 可利 用面积 $3.10 \times 10^{8}$ ha, 对发展畜牧业、保护生物多样 性、保持水土和维护生态平衡有着重要的作用和价 值(谢高地等, 2001)。放牧是草原最普遍和最经济的 利用方式之一, 也是调节草地生产力最有效的管理 方式(刘勇等, 2016)。另一方面, 在对草原生态系统 的众多干扰因素中, 放牧干扰强度最大、频率最高、 干扰时间最长、影响后果最严重(李文龙等, 2010)。 不同的放牧强度对草原生态系统会产生不同的影 响, 过度放牧直接导致植物多样性(宋洁等, 2019)、 土壤动物物种与群落多样性(武崎等, 2016)以及土 壤微生物群落多样性(丁成翔等, 2020)的显著下滑, 导致草原植被退化, 从而加剧土壤荒漠化(李东丽, 2020)。荒漠化的土壤动物群落又通过改变土壤特 性、微生物活动等影响分解过程和生态系统的物质 循环和能量流动(Wardle et al, 2004), 进而影响生物 生存环境, 进一步加剧生物多样性的下滑。

蜘蛛是草原中常见的动物类群之一, 是许多草 原害虫的重要天敌。蜘蛛多样性的变化可以反映土 地利用的变化(Duan et al, 2019a), 以及其他土壤生 物群落的组成、丰度和丰富度的变化(Bell et al, 2001)。蜘蛛在自然生物控制中起着重要作用, 它们 对耕作、播种、收获、施用农药、放牧方式都很敏 感(Freiberg et al, 2019), 常被选作生物多样性指示 类群进行研究。蜘蛛主要有两种捕食方式: 织网型 和游猎型。蜘蛛捕食方式在驱动营养级联和影响生 态系统功能方面具有重要意义(Perkins et al, 2018)。 近年来, 有研究从不同角度探讨了蜘蛛功能群在各 种生境下的多样性, 但对草地蜘蛛的研究较少(周
文婕等, 2019; 胡文浩等, 2020), 而放牧强度对两种 捕食方式蜘蛛多样性的影响也尚不清楚。

本研究在内蒙古赛罕乌拉草原选择5个不同放 牧强度的样地, 分析蜘蛛的群落多样性、分布格局 以及功能群结构的差异, 以及植被高度对蜘蛛多样 性的影响, 探讨放牧强度对蜘蛛群落多样性和功能 群的影响, 以期为草原管理和蜘蛛多样性的保护提 供依据。

\section{材料与方法}

\section{1 研究区域}

研究区域位于内蒙古赤峰市巴林右旗赛罕乌 拉草原 $\left(118^{\circ} 18^{\prime}-118^{\circ} 55^{\prime} \mathrm{E}, 43^{\circ} 59^{\prime}-44^{\circ} 27^{\prime} \mathrm{N}\right)$ 。该地 区是草原向森林、东亚阔叶林向大兴安岭寒温带针 叶林过渡的地带, 属中温带半湿润温寒气候区, 冬 季漫长而寒冷, 夏季短而骤热, 年均降水量 $400 \mathrm{~mm}$ 左右(袁梨等, 2009)。土壤主要以山地黑土、灰黑土、 暗棕壤、暗栗钙土为主(周梅等, 2014)。主要植物为 羊草 (Leymus chinensis)、叉分苶 (Polygonum divaricatum)、灰脉薹草(Carex appendiculata)、我鸟线 委陵菜 (Potentilla anserina) 和寸草臺 (Carex duriuscula)等(张书理等, 2008)。

\section{2 蜘蛛采集及标本鉴定}

根据不同放牧强度选取 5 块样地, 每块样地面 积在 $1 \mathrm{~km}^{2}$ 以上。(1)样地I: 位于二林坝, 由重度放牧 样地包围所形成的一块孤岛, 由灌丛包围, 内部为 草地, 没有牲畜进去取食, 平均植被高度19.31 cm; (2)样地II: 未放牧样地, 位于正沟, 既不打草也不 放牧, 平均植被高度 $27.84 \mathrm{~cm}$; (3)样地III: 轻度放 
牧样地, 位于大东沟, 秋季打草, 偶尔放牧, 约 1 只 羊/ha, 平均植被高度 $25.04 \mathrm{~cm}$; (4)样地IV: 中度放 牧样地, 位于常兴东沟, 秋季打草, 打草后的秋季 和春季放牧, 约4只羊/ha, 平均植被高度 $17.09 \mathrm{~cm}$; (5)样地 V: 重度放牧样地，位于二林坝，约6只羊/ha, 平均植被高度 $4.11 \mathrm{~cm}$ (图1), 样地间隔 $500 \mathrm{~m}$ 以上。 每块样地随机选取 3 个 $2 \mathrm{~m} \times 25 \mathrm{~m}$ 的样方, 相邻样方 间距至少 $100 \mathrm{~m}$ 。

在样地选择时, 取样样方与林地或其他生境的 最近距离均在 $500 \mathrm{~m}$ 以上, 以尽量减少周围生境的 影响。2014年8月, 我们采用扫网法和徒手法从样方 四周边缘向中心逐步进行蜘蛛样本采集。所有取样 均于 9:30 am开始、天气晴朗或微阴、气温 $>15^{\circ} \mathrm{C}$ 、 风速 $<2.5 \mathrm{~m} / \mathrm{s}$ 的条件下进行。在样方内边走边挥网,
每 $1 \mathrm{~s}$ 挥网1次，持续进行 $5 \mathrm{~min}$ 。步行速度大约为 6-7 m/min, 捕虫网网纱 80 目, 网口直径 $40 \mathrm{~cm}$, 网 深 $55 \mathrm{~cm}$, 杆长 $110 \mathrm{~cm}$ 。扫网后, 将样方内的草压倒, 尽可能采集草根、落叶层下、石下等各种微生境中 的蜘蛛, 由两个人前后各采集1次。相同样地内的样 方采集时间相当，但不同样地的样方采集时间随样 地的复杂程度不同而不同: 植被茂盛、蜘蛛个体丰 富的样方采集时间长，超过了 $1 \mathrm{~h}$; 重度放牧样地约 $20 \mathrm{~min}$ 。所有样地都是相同的取样人和取样时间, 并尽量收集到样方内所有的蜘蛛个体。采集的标本 保存于75\%的酒精中带回实验室进行鉴定。成体根 据第二性征(触肢器和外雌器)鉴定到物种水平; 幼 体根据体形、眼的排列、身体上的斑纹等特征，尽 可能鉴定到种，部分幼体只能鉴定到属或科(以属

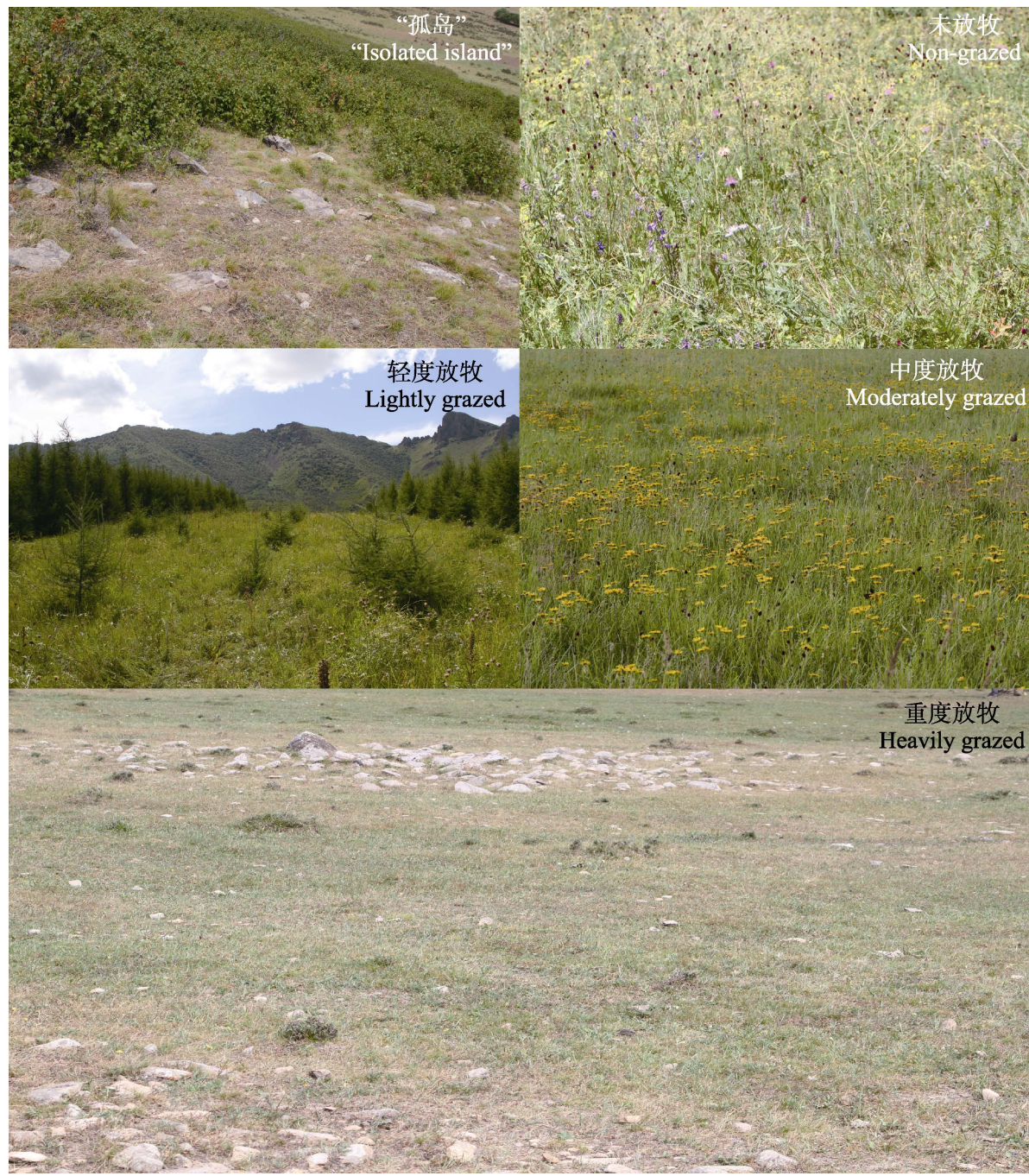

图1 不同放牧强度样地生境照片

Fig. 1 Photos of sample sites 
名或科名 $+\mathrm{sp}$.表示, 如同属或同科有多种类似幼 体, 以sp.1, sp.2, ..., 等表示)。

参考Sanders等(2011，2015)的功能群分类方法， 将蜘蛛分为织网型和游猎型。

\section{3 植被调查}

在每块样地中沿对角线选取 3 块 $50 \mathrm{~cm} \times 50 \mathrm{~cm}$ 的小样方, 记录样方中出现的维管植物物种名和数 量, 测定物种的平均高度, 以样方中所有维管植物 物种的高度加权平均值作为植被群落的平均高度。

\section{4 数据处理}

采用蜘蛛科数、物种数、总个体数、织网型蜘 蛛个体数和游猎型蜘蛛个体数作为蜘蛛多样性的 评价指标, 并将个体数量占该样地个体总数 $10 \%$ 以 上的物种定义为优势种。在ArcGIS 10.2中利用 Mantel I空间自相关分析对各个蜘蛛多样性指标进 行分析, 结果显示各指标在不同样地间均不存在显 著的空间自相关。采用单因素方差分析检验各样地 蜘蛛多样性的差异, 利用LSD检验进行不同放牧强 度下蜘蛛多样性的多重比较。在方差分析前对变量 进行正态性和齐性检验, 不符合正态性和方差齐性 的变量进行对数转换。为了确定不同放牧强度下蜘 蛛群落物种组成的差异, 分别对科级组成、物种组 成、织网型蜘蛛和游猎型蜘蛛物种组成, 基于 Bray-Curtis 指数进行非度量多维标度分析 (non-metric multidimensional scale, NMDS)。进一步 通过相似性分析(analysis of similarities, ANOSIM) 研究不同放牧强度样地的组内及组间差异, 差异显 著性采用999次重复置换检验。最后, 对植被高度和 蜘蛛群落多样性指数进行相关性分析。以上数据分 析使用R 3.6.1中的vegan包和agricolae包。

\section{2 结果}

\section{1 蜘蛛群落组成}

5 个样地共采集到蜘蛛 19 科 79 种 1,112 头, 其中 幼体 744 头, 占比 $66 \%$ (附录1)。逍遥蛛科、蟹蛛科、 狼蛛科和园蛛科的个体数量最多, 分别占总个体数 的 $23.11 \% 、 17.27 \% 、 14.12 \%$ 和 $11.33 \%$ 。不同放牧强 度样地中蜘蛛群落的类群数、优势类群和常见类群 组成不同。样地 I的优势种为娇长逍遥蛛(Tibellus tenellus)、金黄逍遥蛛(Philodromus aureoles)、岛民 花蟹蛛(Xysticus insulicola)和灌木新园蛛(Neoscona adianta), 个体数分别占该样地总数的 $69.59 \%$ 、
$15.67 \% 、 14.75 \%$ 和 $14.29 \%$; 样地II的优势种为朝鲜 狼逍遥蛛(Thanatus coreanus)、岛民花蟹蛛和申氏豹 蛛(Pardosa schenkeli), 个体数分别占该样地总数的 $12.68 \% 、 10.87 \%$ 和 $10.67 \%$; 样地III的优势种为朝鲜 狼逍遥蛛和岛民花蟹蛛, 个体数分别占该样地总数 的 $25.10 \%$ 和 $16.87 \%$; 样地IV的优势种为灌木新园 蛛、印痕叶球蛛(Phylloneta impressa)和岛民花蟹蛛, 个体数分别占该样地总数的 $22.34 \% 、 17.02 \%$ 和 $17.02 \%$; 样地 $\mathrm{V}$ 的优势种为星豹蛛 (Pardosa astrigera)、岛民花蟹蛛和肥腹蛛属一种 (Steatoda sp.), 个体数分别占该样地总数的 $21.43 \% 、 14.29 \%$ 和 $10.00 \%$ 。蜘蛛丰富度最高的样地为未放牧的样地 II, 采集到 497 个个体。而丰富度最低的是重度放牧 的样地 $\mathrm{V}$, 仅采集到 70 个个体。

\section{2 蜘蛛群落多样性差异分析}

蜘蛛科数、物种数、个体数、织网型蜘蛛数、 游猎型蜘蛛数均随着放牧强度的增加而减少, 呈负 相关, 而且不同放牧强度下各类多样性指标均有显 著性差异(图2)。其中未放牧样地(II)的蜘蛛物种数、 个体数、织网型蜘蛛数和游猎型蜘蛛数都极显著高 于中度放牧样地(IV)和重度放牧样地 $(\mathrm{V})$ 。轻度放牧 样地(III)、被重度放牧包围的样地(I)和未放牧样地 (II)的物种数、蜘蛛个体数、织网型蜘蛛数和游猎型 蜘蛛数差异都不明显。中度放牧样地(IV)和重度放 牧样地 $(\mathrm{V})$ 间5种多样性指标的差异也不明显。除重 度放牧样地 $(\mathrm{V})$, 其他四个样地间的织网型蜘蛛个 体数差异均不显著。而游猎型蜘蛛在未放牧样地(II) 与中度放牧(IV)和重度放牧样地(V)间差异显著。对 两种功能群中捕获数量较多的科——狼蛛科、跳蛛 科、园蛛科等的分析发现，只有园蛛科的物种数和 个体数在不同放牧强度间存在显著差异(图3), 而狼 蛛科和跳蛛科的物种数和个体数差异都不显著。

\section{3 蜘蛛群落及功能群相似性分析}

通过NMDS分析结果可知(图4), 不同放牧强度 样地的 3 个样方各自聚集在一起，说明不同放牧强 度下蜘蛛群落组成具有一定差异。从总体上看, “孤 岛”样地、未放牧样地和轻度放牧样地的蜘蛛群落 更为接近，与后两个放牧梯度的蜘蛛群落有较显著 的差异。NMDS第一轴主要代表了放牧强度的差异, 各样地蜘蛛分类群在科一级上的组成差异在放牧 强度越接近的情况下越小, 被重度放牧区域所包围 的样地 $\mathrm{I}$ 与未放牧和轻度放牧样地之间更为相似, 

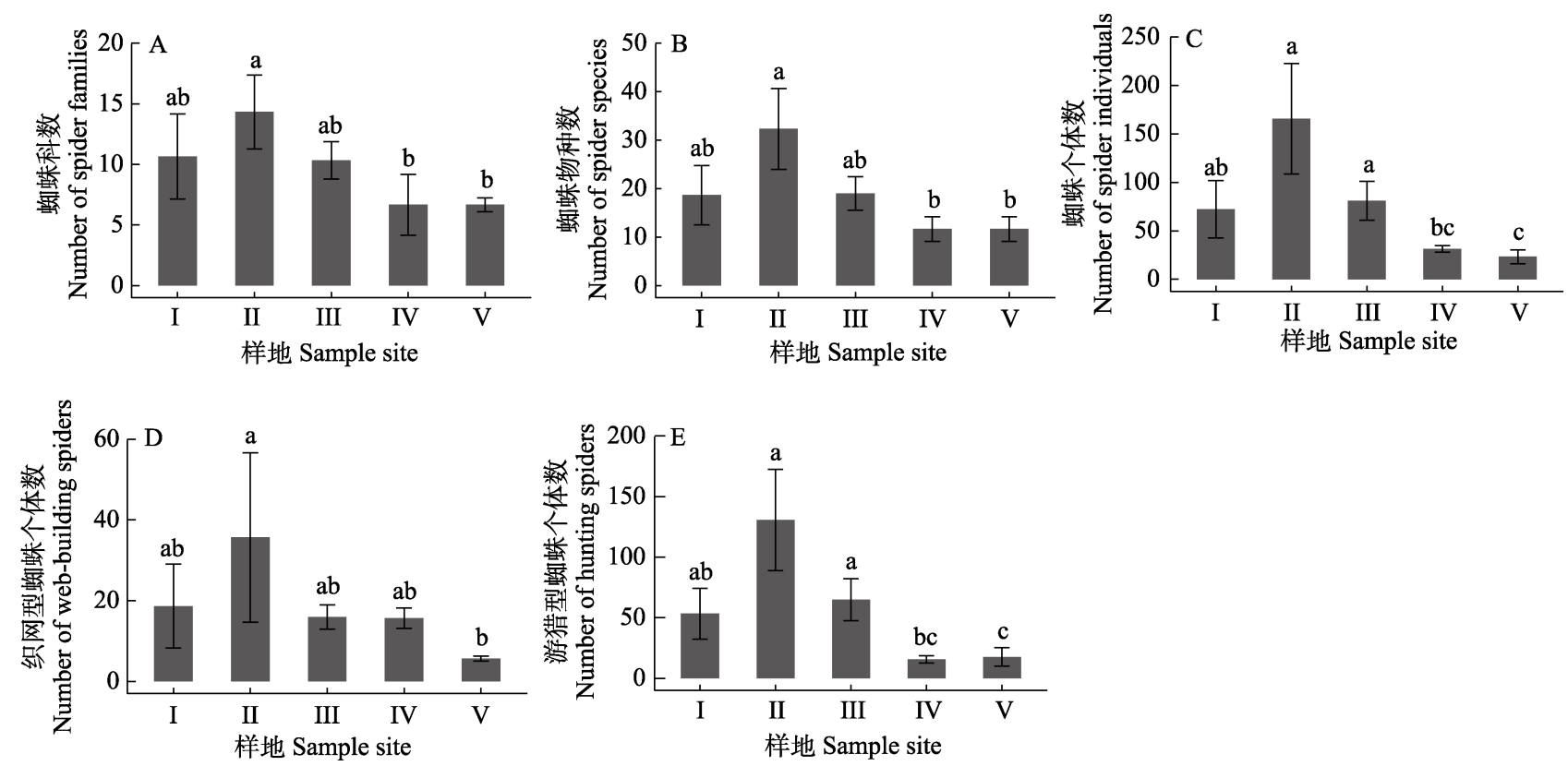

图2 不同放牧强度下蜘蛛的科数 $(\mathrm{A})$ 、种数 $(\mathrm{B})$ 、个体数 $(\mathrm{C})$ 、织网型蜘蛛个体数 $(\mathrm{D})$ 和游猎型蜘蛛个体数 $(\mathrm{E})$ (平均值 \pm 标准 误)。不同小写字母表示不同生境间差异显著 $(P<0.05) 。$ I: “孤岛”; II: 未放牧样地; III: 轻度放牧样地; IV: 中度放牧样地; V: 重度放牧样地。

Fig. 2 The number of spider families (A), species (B), individuals (C), web-building spiders (D) and hunting spiders (E) of different grazing intensities (Mean \pm SE). Different letters show significant differences among different sample sites $(P<0.05)$. I, "Isolated island”; II, Non-grazed; III, Lightly grazed; IV, Moderately grazed; V, Heavily grazed.
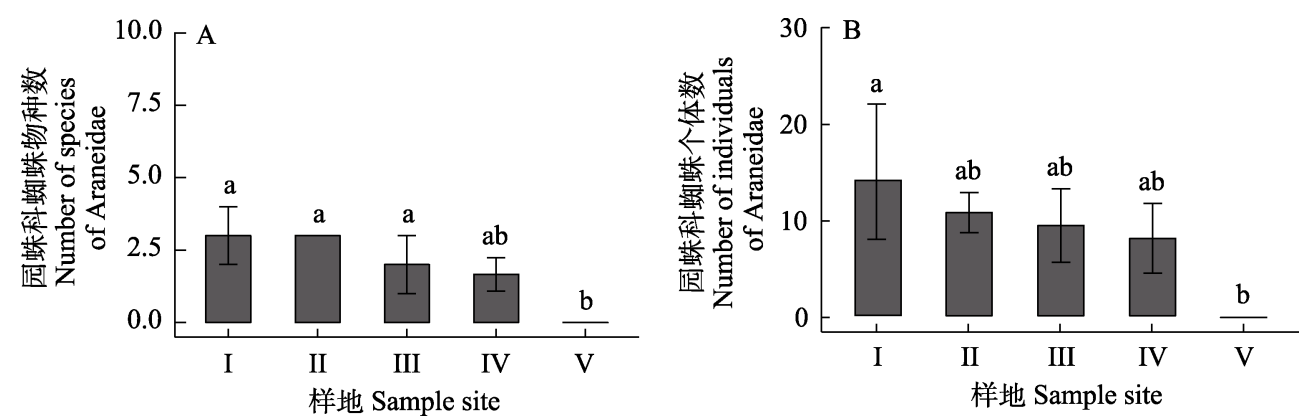

图3 不同放牧强度下园蛛科蜘蛛物种数(A)和个体数(B) (平均值 \pm 标准误)。不同字母表示不同生境间的差异显著 $(\boldsymbol{P}<$ 0.05)。II: “孤岛”; II: 未放牧样地; III: 轻度放牧样地; IV: 中度放牧样地; V: 重度放牧样地。

Fig. 3 The number of species (A) and individuals (B) of Araneidae under different grazing intensities (Mean $\pm \mathrm{SE}$ ). Different letters show significant differences among different sample sites $(P<0.05)$. I, "isolated island"; II, Non-grazed; III, Lightly grazed; IV, Moderately grazed; V, Heavily grazed.

而与中度放牧样地和重度放牧样地差异更大(图 4A)。在物种一级上, 蜘蛛类群的物种组成呈现更为 明显的梯度变化, 放牧强度越低, 物种组成和未放 牧样地(II)越相近(图4B)。而织网型蜘蛛和游猎型蜘 蛛的物种组成在孤岛(I)、未放牧(II)和轻度放牧(III) 3 个样地间更为相近, 与重度放牧样地 $(\mathrm{V})$ 差异较 大。此外, 相似性分析(ANOSIM) 表明蜘蛛群落在科 级 $(\mathrm{R}=0.726, P<0.001)$ 、种级 $(\mathrm{R}=0.791, P<$ $0.001)$ 、织网型 $(\mathrm{R}=0.599, P<0.001)$ 和游猎型 $(\mathrm{R}=$
$0.721, P<0.001)$ 四个指标上，不同放牧强度样地中 的组间差异显著大于组内差异。

\section{4 植被高度与蜘蛛多样性的相关性分析}

相关分析发现植被高度与蜘蛛物种数、蜘蛛个 体数、织网型蜘蛛个体数和游猎型蜘蛛个体数显著 正相关 $(P<0.05)$, 而与蜘蛛科数无显著的正相关关 系 $(P=0.056)$ (图5)。此外, 对采集标本中数量较多 的科与植被高度的相关性分析结果表明: 植被高度 与蟹蛛科和逍遥蛛科呈极显著正相关 $(P<0.001)$, 


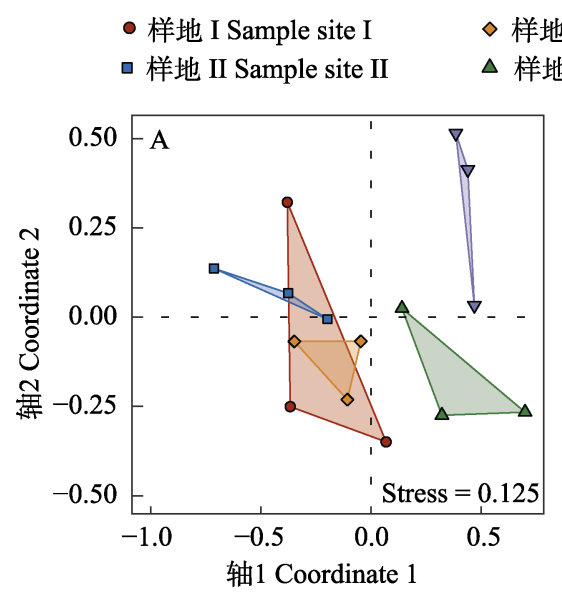

$\diamond$ 样地 III Sample site III

- 样地 I Sample site I

样地 IV Sample site IV
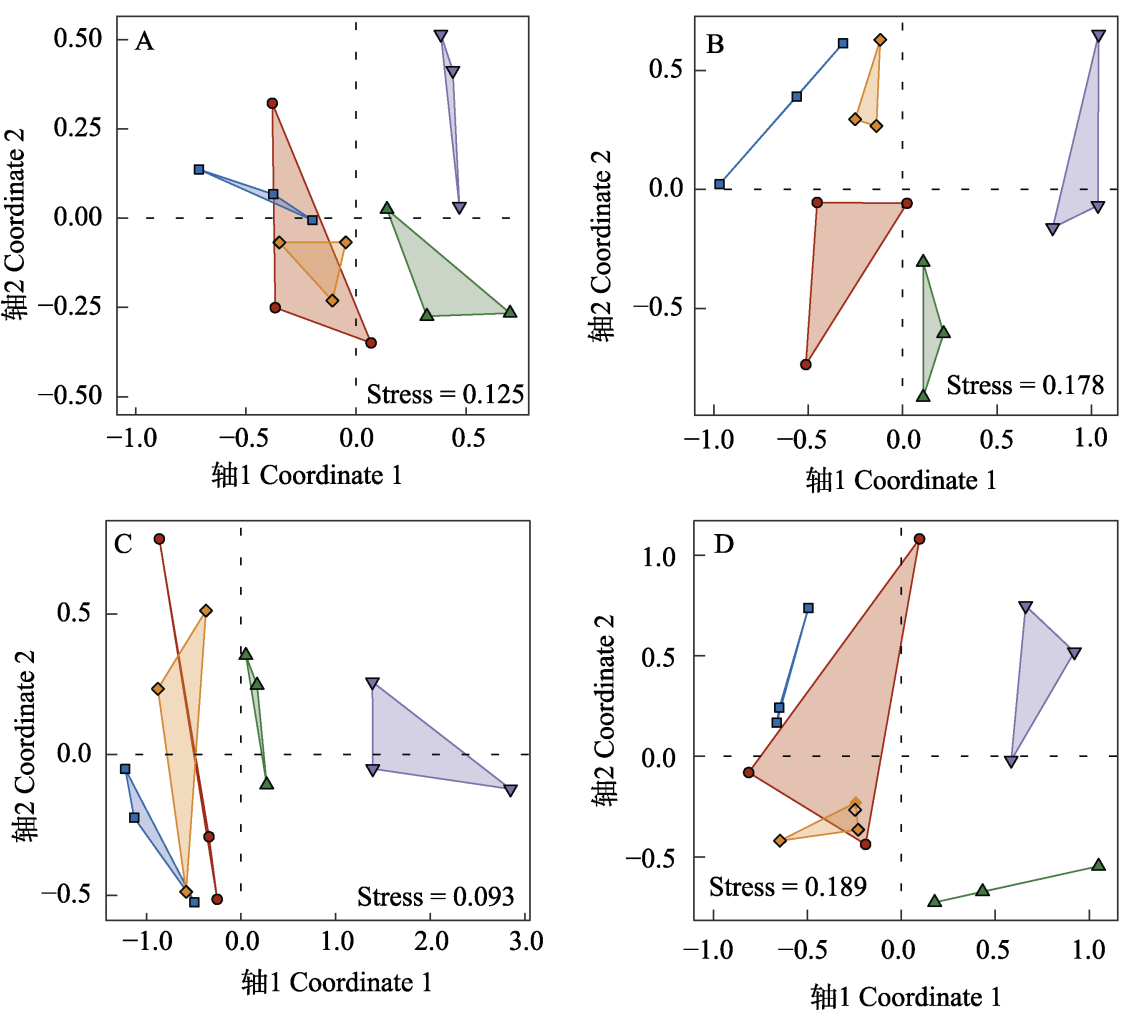

图4 基于NMDS的不同放牧强度样地的蜘蛛群落。A: 科级; B: 种级; C: 织网型蜘蛛; D: 游猎型蜘蛛。I: “孤岛”; II: 未放 牧样地; III: 轻度放牧样地; IV: 中度放牧样地; V: 重度放牧样地。

Fig. 4 Community composition of sample plots under different grazing intensities by NMDS analysis。A, Family; B, Species; C, Web-building spiders; D, Hunting spiders. I, “Isolated island”; II, Non-grazed; III, Lightly grazed; IV, Moderately grazed; V, Heavily grazed.

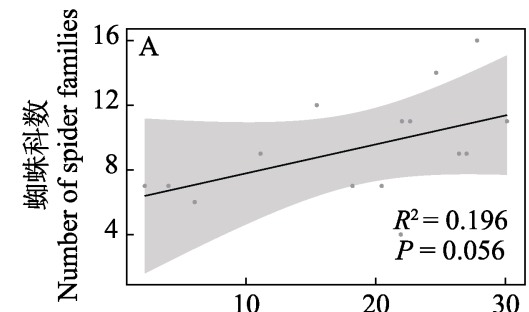

植被高度 Vegetation height $(\mathrm{cm})$

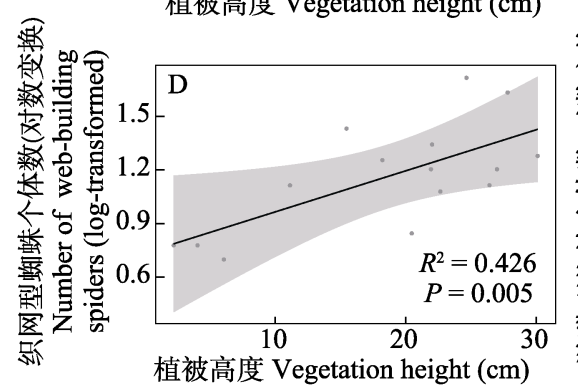

植被高度 Vegetation height $(\mathrm{cm})$

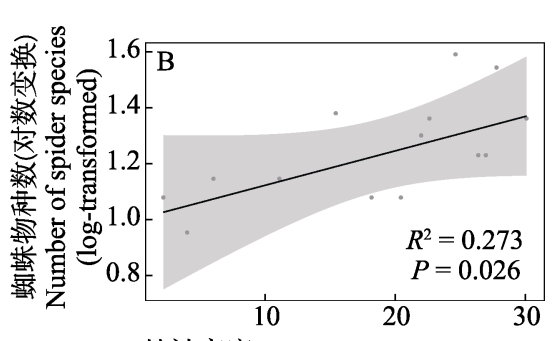

植被高度 Vegetation height $(\mathrm{cm})$

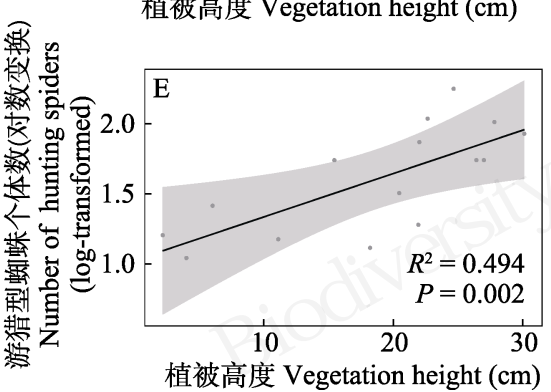

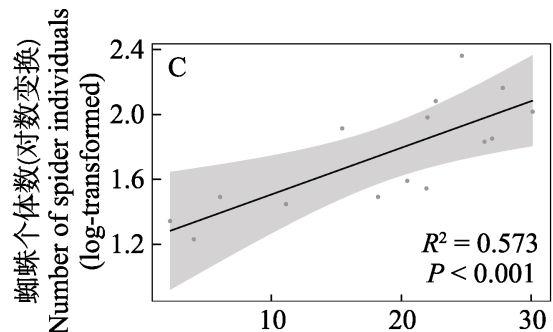

植被高度 Vegetation height $(\mathrm{cm})$

图5 蜘蛛多样性与植被高度的相关性拟合

Fig. 5 Correlation fitting of spider biodiversity and vegetation height 

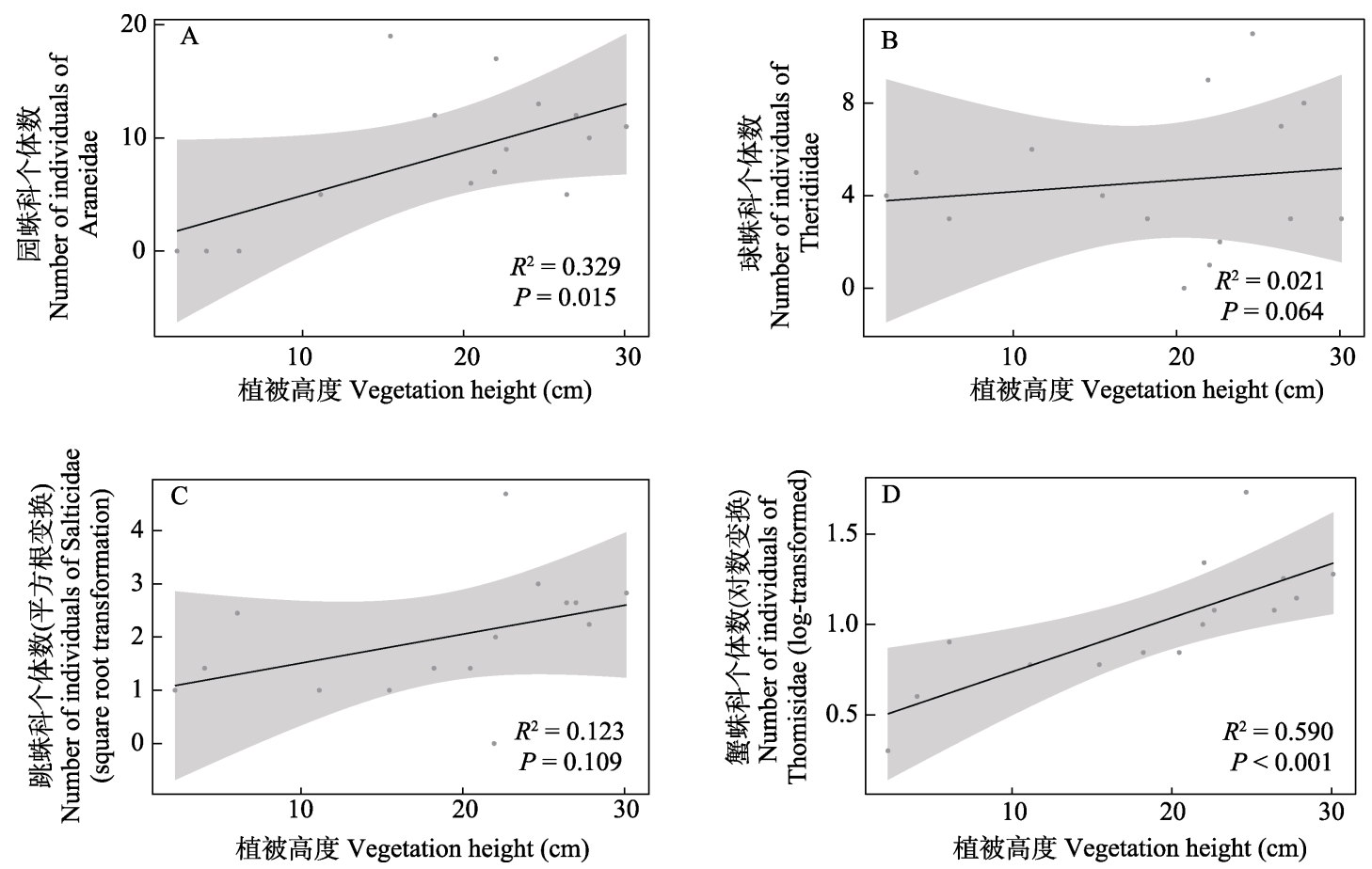

图6 蜘蛛各科个体数与植被高度的相关性拟合

Fig. 6 Correlation fitting of number of spider families and vegetation height

与园蛛科呈显著正相关 $(P=0.015)$, 与球蛛科、跳蛛 科和狼蛛科不存在显著的相关性 $(P=0.604 ; P=$ $0.109 ; P=0.367$ ) (图6)。草原植被高度与蜘蛛多样 性相关性比较显著, 总体上是植被高度越高的样地, 蜘蛛科数、物种数、个体数以及织网蜘蛛个体数和 游猎蜘蛛个体数也更多。

\section{3 讨论}

\section{1 放牧强度对蜘蛛多样性的影响}

与其他研究 (Gibson et al, 1992; Řezáč \& Heneberg, 2019)类似, 我们也发现重度放牧样地(V) 与未放牧样地(II)之间的蜘蛛科数、物种数、个体数、 织网型蜘蛛数和游猎型蜘蛛数均有着显著差异, 符 合资源异质性与生产力假说(resource-heterogeneity and resource-productivity hypotheses) (Siemann, 1998; Waide et al, 1999)。上述样地的植被特征差异明显, 重度放牧样地牧草的高度仅有 $4 \mathrm{~cm}$ 左右, 而未放牧 样地牧草高度超过 $20 \mathrm{~cm}$ 。牧草高度越高, 垂直结构 越复杂, 空间异质性越高, 为蜘蛛提供的隐蔽场所 就越多, 蜘蛛多样性也更为丰富(图5) (Dennis et al, 2001; Horváth et al, 2009)。我们的研究结果也印证 了植被高度对蜘蛛物种数、个体数、织网型蜘蛛、
游猎型蜘蛛和常见科的个体数都有显著的影响。一 种可能的原因是上行效应(bottom-up effect)。放牧通 过破坏植物与昆虫的相互作用并减少了地上无脊 椎动物栖息地的空间大小和复杂性来影响昆虫群 落, 如直翅目、鳞翅目、膜翅目等, 它们均为蜘蛛 的主要猎物类群(Kruess \& Tscharntke, 2002)。

\section{2 放牧强度对不同功能群蜘蛛的影响}

织网型蜘蛛需要环境中有适宜的物理结构支 持才能织网捕食(Lubin, 1978), 因此, 植被高度相 对较高的样地(II、III、IV)的织网型蜘蛛多样性差异 不明显, 而植被高度仅 $5 \mathrm{~cm}$ 的样地(V)却难见其踪 影(图2D)。而且, 由于重度放牧区域牲畜频繁地践 踏, 导致在地面织漏斗网的漏斗蛛科也难以生存 (Warui et al, 2005)。本研究中织网型蜘蛛的多样性 及组成差异对于不同放牧强度的响应相比于游猎 型蜘蛛更小。这可能是由于游猎型蜘蛛受植被结构 的影响相比织网型蜘蛛要小, 其多样性减少更可能 是由于猎物的可获得性减少导致。游猎型蜘蛛活动 能力强, 消耗能量快, 需要频繁进食, 当重度放牧 样地的食物资源不足时, 便会开始迁移。而织网型 蜘蛛几乎均为“坐等型”捕食者, 能量消耗慢, 受食 物资源影响相对较小(Sanders et al, 2011)。植被高度 
差异明显的样地 $\mathrm{IV}$ 与样地 $\mathrm{V}$ 之间蜘蛛多样性差异不 明显, 这可能是由于重度放牧会增加耐受能力较强 蜘蛛的多样性。例如, 狼蛛是蜘蛛中的拓荒者, 其 耐受能力非常强。我们在样地 $\mathrm{V}$ 发现了 5 种狼蛛 22 个个体; 而在样地IV只发现了 3 种狼蛛 12 个个体。分 别占到各自蜘蛛群落物种数及个体数的 $22.73 \%$ 、 $30.99 \%$ 和 $14.29 \% 、 12.77 \%$ 。虽然样地 $\mathrm{V}$ 织网型蜘蛛 较少, 但狼蛛及其他耐受能力强的物种的种类和个 体的增加使两个样地的蜘蛛多样性差异不大。相对 于对放牧强度响应不显著的狼蛛科和跳蛛科, 园蛛 科的物种数和个体数在不同放牧强度样地间差异 显著(图4)。这可能是由于狼蛛和跳蛛以游猎型为主, 主要在地表活动, 而园蛛科主要在植物上织网取食, 因此对于植物结构具有更为强烈的需求(Bell et al, 2001; Schmidt et al, 2008)。而放牧强度对于样地的 影响主要就是体现在植物群落上, 植被高度越高, 可供园蛛织网的空间就越多, 园蛛科个体数也随之 增多。狼蛛和跳蛛由于更强的活动能力, 可以尽量 降低过度放牧的负面影响, 因而植被高度和狼蛛 科、跳蛛科个体数之间没有显著的相关性(图6C) (Duan et al, 2019a)。因此, 织网型蜘蛛, 特别是园蛛 科, 更适合作为重度放牧的指示类群。

值得注意的是, 本研究观察到球蛛科与植被高 度呈不显著正相关, 这可能是由于板隅拟肥腹蛛 (Parasteatoda tabulata)、温室拟肥腹蛛 $(P$. tepidariorum) 和拟肥腹蛛属一种(Parasteatoda sp.) 虽为织网型蜘蛛, 但却偏好在大石块下织网, 因此 对植被结构的依赖性不高。而逍遥蛛科和蟹蛛科与 植被高度呈极显著正相关(图6), 这可由逍遥蛛科和 蟹蛛科的习性及多捕食者效应(multiple predators effect) 解释。主动出击的游猎型蜘蛛(如跳蛛、狼蛛 等)多栖息于植被的中下层, 而逍遥蛛科及蟹蛛科 等依靠伏击猎物捕食的蜘蛛倾向于占领植被的冠 层(Barton \& Schmitz, 2009)。当放牧强度增加, 植被 高度降低, 迫使它们的栖息地下移, 从而两者的生 境重合。实验模拟(Schmitz, 2007)表明, 这种情况会 导致种间侵略, 因为捕食者在有限的空间中争夺和 保卫狩猎区域。主动出击的蜘蛛获取资源的能力更 强, 受到植被高度降低的影响较小, 抑制了逍遥蛛 科及蟹蛛科的种群数量。因而植被高度对逍遥蛛科 及蟹蛛科的显著影响更可能是间接效应(Barton \& Schmitz, 2009)。

\section{3 残存生境对蜘蛛多样性的影响}

样地 $\mathrm{I}$ 位于重度放牧区域之中, 是由重度放牧 草原包围形成的一片生境孤岛, 四周为一片灌木林, 从而与重度放牧样地隔开。茂盛的灌木丛阻碍了牛 羊的踩踏, 使这片孤岛未受放牧的影响。大多数蜘 蛛在幼年期都具有飞航的能力, 且飞航能力从几米 到几千公里不等, 从而使其可以逃离原生生境的不 利环境, 在新的适宜生境快速建立种群(Bell et al, 2005; Pfister et al, 2015; Duan et al, 2019b)。样地I的 物种组成与未放牧样地和轻度放牧样地更接近, 却 与周边的重度放牧样地差异很大, 可能是集约化的 土地利用强度迫使蜘蛛进行迁徙和寻找避难所, 使 周边重度放牧草地中的蜘蛛群落向样地转移, 导 致样地I保持着周边样地未放牧时的多样性, 因而 与未放牧样地物种组成更接近(Rand et al, 2006)。可 见残存生境对蜘蛛保护具有重要意义。

草原蜘蛛群落的多样性及功能群结构与生境 类型及土地利用强度密切相关, 更高的空间及资源 异质性能有效提高蜘蛛的多样性。织网型和游猎型 两种不同捕食方式的蜘蛛占据不同的生态位, 并起 到连接草原生态系统不同食物网的重要作用。因此, 减少放牧强度(放牧持续时间和牲畜密度等), 维持 植被群落复杂的垂直结构, 有利于蜘蛛多样性的保 护, 考虑到蜘蛛的捕食性, 可以具有较好的虫害生 物控制潜力, 对草原的可持续发展和利用有着重要 意义。

\section{ORCID}

张宇 (D) https://orcid.org/0000-0003-0663-9852

王露雨 (D) https://orcid.org/0000-0002-5250-3473

段美春 (10 https://orcid.org/0000-0002-9061-2654

张志升 (D) https://orcid.org/0000-0002-9304-1789

\section{参考文献}

Barton BT, Schmitz OJ (2009) Experimental warming transforms multiple predator effects in a grassland food web. Ecology Letters, 12, 1317-1325.

Bell JR, Bohan DA, Shaw EM, Weyman GS (2005) Ballooning dispersal using silk: World fauna, phylogenies, genetics and models. Bulletin of Entomological Research, 95, 69-114.

Bell JR, Wheater CP, Cullen WR (2001) The implications of grassland and heathland management for the conservation of spider communities: A review. Journal of Zoology, 255, 377-387.

Dennis P, Young MR, Bentley C (2001) The effects of varied 
grazing management on epigeal spiders, harvestmen and pseudoscorpions of Nardus stricta grassland in upland Scotland. Agriculture, Ecosystems \& Environment, 86, 39-57.

Ding CX, Yang XX, Dong QM (2020) Effects of grazing patterns on vegetation, soil and microbial community in alpine grassland of Qinghai-Tibetan Plateau. Acta Agrestia Sinica, 28, 159-169. (in Chinese with English abstract) [丁 成翔, 杨晓霞, 董全民 (2020) 青藏高原高寒草原放牧方 式对植被、土壤及微生物群落的影响. 草地学报, 28, 159-169.]

Duan MC, Hu WH, Liu YH, Yu ZR, Li X, Wu PL, Zhang F, Shi HL, Baudry J (2019a) The influence of landscape alterations on changes in ground beetle (Carabidae) and spider (Araneae) functional groups between 1995 and 2013 in an urban fringe of China. Science of the Total Environment, 689, 516-525.

Duan MC, Liu YH, Li X, Wu PL, Hu WH, Zhang F, Shi HL, Yu ZR, Baudry J (2019b) Effect of present and past landscape structures on the species richness and composition of ground beetles (Coleoptera: Carabidae) and spiders (Araneae) in a dynamic landscape. Landscape and Urban Planning, 192, 103649.

Freiberg JA, de Sales Dambros C, Rodrigues ENL, Teixeira RA, Vieira ÂDHN, de Almeida HS, de Faccio Carvalho PC, Jacques RJS (2019) Increased grazing intensity in pastures reduces the abundance and richness of ground spiders in an integrated crop-livestock system. Agronomy for Sustainable Development, 40, 1-10.

Gibson CWD, Hambler C, Brown VK (1992) Changes in spider (Araneae) assemblages in relation to succession and grazing management. Journal of Applied Ecology, 29, 132-142.

Horváth R, Magura T, Szinetár C, Tóthmérész B (2009) Spiders are not less diverse in small and isolated grasslands, but less diverse in overgrazed grasslands: A field study (East Hungary, Nyírség). Agriculture, Ecosystems \& Environment, 130, 16-22.

Hu WH, Duan MC, Na SH, Zhang F, Yu ZR (2020) Spider diversity and community characteristics in cropland and two kinds of recovery habitats in Bashang area, China. Chinese Journal of Applied Ecology, 31, 643-650. (in Chinese with English abstract) [胡文浩, 段美春, 那书豪, 张锋, 宇振荣 (2020) 坝上地区农田及两种恢复生境中蜘蛛多样性与群 落特征. 应用生态学报, 31, 643-650.]

Kruess A, Tscharntke T (2002) Grazing intensity and the diversity of grasshoppers, butterflies, and trap-nesting bees and wasps. Conservation Biology, 16, 1570-1580.

Li DL (2020) On the causes of grassland desertification and the countermeasures. Forestry Science and Technology Information, 52(1), 16-18. (in Chinese with English abstract) [李东丽 (2020) 草原荒漠化的成因及防治对策. 林业科技情报, 52(1), 16-18.]
Li WL, Su M, Li ZZ (2010) Analysis of AHP strategic decisions for the ecological risk of grazing system and management decisions in an alpine meadow at Gannan, Gansu. Acta Prataculturae Sinica, 19, 22-28. (in Chinese with English abstract) [李文龙, 苏敏, 李自珍 (2010) 甘 南高寒草地放牧系统生态风险的AHP决策分析及管理对 策. 草业学报, 19, 22-28.]

Liu Y, Zhang YW, Nan ZB, Duan TY (2016) Progress of research into the effects of native grassland management practices on plant disease. Acta Ecologica Sinica, 36, 4211-4220. (in Chinese with English abstract) [刘勇, 张雅 雯, 南志标, 段廷玉 (2016) 天然草地管理措施对植物病 害的影响研究进展. 生态学报, 36, 4211-4220.]

Lubin YD (1978) Seasonal abundance and diversity of web-building spiders in relation to habitat structure on Barro Colorado Island, Panama. Journal of Arachnology, 6, 31-51.

Perkins MJ, Inger R, Bearhop S, Sanders D (2018) Multichannel feeding by spider functional groups is driven by feeding strategies and resource availability. Oikos, 127, 23-33.

Pfister SC, Schäfer RB, Schirmel J, Entling MH (2015) Effects of hedgerows and riparian margins on aerial web-building spiders in cereal fields. Journal of Arachnology, 43, 400-405.

Rand TA, Tylianakis JM, Tscharntke T (2006) Spillover edge effects: The dispersal of agriculturally subsidized insect natural enemies into adjacent natural habitats. Ecology Letters, 9, 603-614.

Řezáč M, Heneberg P (2019) Grazing as a conservation management approach leads to a reduction in spider species richness and abundance in acidophilous steppic grasslands on andesite bedrock. Journal of Insect Conservation, 23, 777-783.

Sanders D, Schaefer M, Platner C, Griffiths GJK (2011) Intraguild interactions among generalist predator functional groups drive impact on herbivore and decomposer prey. Oikos, 120, 418-426.

Sanders D, Vogel E, Knop E (2015) Individual and species-specific traits explain niche size and functional role in spiders as generalist predators. Journal of Animal Ecology, 84, 134-142.

Schmidt MH, Thies C, Nentwig W, Tscharntke T (2008) Contrasting responses of arable spiders to the landscape matrix at different spatial scales. Journal of Biogeography, 35, 157-166.

Schmitz OJ (2007) Predator diversity and trophic interactions. Ecology, 88, 2415-2426.

Siemann E (1998) Experimental tests of effects of plant productivity and diversity on grassland arthropod diversity. Ecology, 79, 2057-2070.

Song J, Wang FG, Wen L, Wang LX, Li JL, Wu SN, Xu ZC (2019) Effects of grazing on plant diversity and soil nutrients in typical temperate steppe. Acta Agrestia Sinica, 
27, 1694-1701. (in Chinese with English abstract) [宋洁, 王凤歌, 温璐, 王立新, 李金雷, 武胜男, 徐智超 (2019) 放牧对温带典型草原植物物种多样性及土壤养分的影响. 草地学报, 27, 1694-1701.]

Waide RB, Willig MR, Steiner CF, Mittelbach G, Gough L, Dodson SI, Juday GP, Parmenter R (1999) The relationship between productivity and species richness. Annual Review of Ecology and Systematics, 30, 257-300.

Wardle DA, Bardgett RD, Klironomos JN, Setälä H, van der Putten WH, Wall DH (2004) Ecological linkages between aboveground and belowground biota. Science, 304, 1629-1633.

Warui CM, Villet MH, Young TP, Jocqué R (2005) Influence of grazing by large mammals on the spider community of a Kenyan savanna biome. Journal of Arachnology, 33, 269-279.

Wu Q, Wu PF, Wang Q, Wen YL, Gao YM, Zhang RZ, Long W (2016) Effects of grazing intensity on the community structure and diversity of different soil fauna in alpine meadow. Scientia Agricultura Sinica, 49, 1826-1834. (in Chinese with English abstract) [武崎, 吴鹏飞, 王群, 文勇 立, 高艳美, 张荣芝, 龙伟 (2016) 放牧强度对高寒草地 不同类群土壤动物的群落结构和多样性的影响. 中国农 业科学, 49, 1826-1834.]

Xie GD, Zhang YL, Lu CX, Zheng D, Cheng SK (2001) Study on valuation of rangeland ecosystem services of China. Journal of Natural Resources, 16, 47-53. (in Chinese with English abstract) [谢高地, 张钇锂, 鲁春霞, 郑度, 成升鬼 (2001) 中国自然草地生态系统服务价值. 自然资源学报, 16, 47-53.]

Yuan L, Dai K, Bao WD, Yu PL (2009) Field survey on amphibians and reptiles in Saihanwula Nature Reserve, Inner Mongolia. Sichuan Journal of Zoology, 28, 283-285. (in Chinese with English abstract) [袁梨, 戴鬼, 鲍伟东, 蔚培龙 (2009) 内蒙古赛罕乌拉国家级自然保护区两栖 爬行动物调查. 四川动物, 28, 283-285.]

Zhang SL, Yong WY, Yang YX, Lu ZX, Zhang F, Li GL (2008) Study on the vegetation classification in Saihanwula Nature Reserve. Journal of Inner Mongolia University (Natural Science Edition), 39, 74-79. (in Chinese with English abstract) [张书理, 雍伟义, 杨永昕, 卢朝霞, 张 帆, 李桂林 (2008) 赛罕乌拉自然保护区植被分类研究. 内蒙古大学学报(自然科学版), 39, 74-79.]

Zhou M, Zeng N, Zhao PW, Wei JS, Li P, Zhai MY (2014) Evaluation on the Betula platyphylla forest ecosystem service values in southern Daxing'anling. Forest Resources Management, (1), 120-126. (in Chinese with English abstract) [周梅, 曾楠, 赵鹏武, 魏江生, 李攀, 翟明瑶 (2014) 大兴安岭南段白桦次生林生态系统服务价值评 估. 林业资源管理, (1), 120-126.]

Zhou WJ, Zhong L, Huang JL, Xie YY, Luo YY (2019) Species diversity, functional group structure, and influencing factors of spider community in fragmented landscape of Thousand Island Lake. Acta Ecologica Sinica, 39, 2226-2236. (in Chinese with English abstract) [周文婕, 仲磊, 黄杰灵, 谢央央, 罗媛媛 (2019) 千岛湖片段化景 观中蜘蛛群落物种多样性和功能群结构及其影响因素. 生态学报, 39, 2226-2236.]

(责任编委: 吴东辉 责任编辑: 问文杰)

\section{附录 Supplementary Material}

附录1 各样地蜘蛛名录

Appendix 1 List of spider species of each sample plot

https://www.biodiversity-science.net/fileup/PDF/2020214-1.pdf 
张宇, 王露雨, 向昌林, 段美春, 张志升 (2021) 不同放牧强度对赛罕乌拉草原蜘蛛多样性的影响. 生物多样性, 29, 467-476. http://www.biodiversity-science.net/CN/10.17520/biods.2020214

附录1 各样地蜘蛛名录

Appendix 1 List of spider species of each sample plot

个体数量 Number of individuals

\begin{tabular}{|c|c|c|c|c|c|c|}
\hline \multirow{3}{*}{ 物种 Species } & & & & & & \multirow{3}{*}{$\begin{array}{l}\text { 捕食方式 } \\
\text { Foraging type }\end{array}$} \\
\hline & 样地 I & 样地II & 样地 III & 样地 IV & 样地V & \\
\hline & $\begin{array}{l}\text { Sample } \\
\text { site I }\end{array}$ & $\begin{array}{l}\text { Sample } \\
\text { site II }\end{array}$ & $\begin{array}{l}\text { Sample site } \\
\text { III }\end{array}$ & $\begin{array}{l}\text { Sample site } \\
\text { IV }\end{array}$ & $\begin{array}{l}\text { Sample } \\
\text { site V }\end{array}$ & \\
\hline
\end{tabular}

漏斗蛛科 Agelenidae

迷宫漏斗蛛 Agelena labyrinthica (Clerck, 1757)

园蛛科 Araneidae

帕氏尖蛛 Aculepeira packardi (Thorell, 1875)

\section{红螯蛛科 Cheiracanthiidae}

绿色红螯蛛 Cheiracanthium virescens Sundevall, 1833

\section{管巢蛛科 Clubionidae}

褐管巢蛛 Clubiona neglecta O. P. -Cambridge, $1862 \quad 1$

管巢蛛属一种 Clubiona sp.

\section{卷叶蛛科 Dictynidae}

\section{平腹蛛科 Gnaphosidae}

掠蛛属一种 Drassodes $\mathrm{sp}$ 


\begin{tabular}{|c|c|c|c|c|c|c|}
\hline \multirow{2}{*}{ 物种 Species } & \multicolumn{5}{|c|}{ 个体数量 Number of individuals } & \multirow{2}{*}{$\begin{array}{l}\text { 捕食方式 } \\
\text { Foraging type }\end{array}$} \\
\hline & $\begin{array}{l}\text { 样地 I } \\
\text { Sample } \\
\text { site I }\end{array}$ & $\begin{array}{l}\text { 样地II } \\
\text { Sample } \\
\text { site II }\end{array}$ & $\begin{array}{l}\text { 样地 III } \\
\text { Sample site } \\
\text { III }\end{array}$ & $\begin{array}{l}\text { 样地 IV } \\
\text { Sample site } \\
\text { IV }\end{array}$ & $\begin{array}{l}\text { 样地V } \\
\text { Sample } \\
\text { site V }\end{array}$ & \\
\hline 狂蛛属一种 Zelotes sp.3 & & & 1 & & & 游猎型 \\
\hline \multicolumn{7}{|l|}{ 皿蛛科 Linyphiidae } \\
\hline 斑血蛛属一种 Abiskoa sp. & & & & 4 & 3 & 织网型 \\
\hline 指丘血蛛 Agyneta dactylis (Tao, Li \& Zhu, 1995) & & & & & 1 & 织网型 \\
\hline 斑血盖蛛 Allomengea dentisetis (Grube, 1861) & & 1 & & & & 织网型 \\
\hline 齿肢微蛛 Erigone dentipalpis (Wider, 1834) & & & & & 1 & 织网型 \\
\hline 三角弗蛛 Floronia bucculenta (Clerck, 1757) & 2 & 27 & 2 & & & 织网型 \\
\hline 卡氏盖蛛 Neriene cavaleriei (Schenkel, 1963) & & 5 & & & & 织网型 \\
\hline 明显盖蛛 Neriene emphana (Walckenaer, 1842) & 1 & & 1 & & & 织网型 \\
\hline 皿蛛科一种 Linyphiidae sp.1 & 2 & 2 & 1 & & & 织网型 \\
\hline 皿蛛科一种 Linyphiidae sp.2 & & 2 & & & & 织网型 \\
\hline 皿蛛科一种 Linyphiidae sp.3 & & 3 & & & & 织网型 \\
\hline \multicolumn{7}{|l|}{ 拟态蛛科 Mimetidae } \\
\hline 诧蛛属一种 Ermetus sp. & & 2 & 13 & & & 游猎型 \\
\hline \multicolumn{7}{|l|}{ 光灰蛛科 Liocranidae } \\
\hline 蒙古田野蛛 Agrocea mongolica Schenkel, 1936 & & 3 & & & & 游猎型 \\
\hline \multicolumn{7}{|l|}{ 狼蛛科 Lycosidae } \\
\hline 白纹舞蛛 Alopecosa albostriata (Grube, 1861) & 4 & 1 & & 9 & 2 & 游猎型 \\
\hline 耳毛舞蛛 Alopecosa auripilosa (Schenkel, 1953) & 3 & 2 & & & & 游猎型 \\
\hline 铸形舞蛛 Alopecosa cuneata (Clerck, 1757) & 2 & & & & & 游猎型 \\
\hline 利氏舞蛛 Alopecosa licenti (Schenkel, 1953) & 1 & & 2 & 2 & 1 & 游猎型 \\
\hline 舞蛛属一种 Alopecosa sp. & 6 & 8 & & 1 & & 游猎型 \\
\hline 田园豹蛛 Pardosa agrestis (Westring, 1861) & & 10 & & & & 游猎型 \\
\hline 星豹蛛 Pardosa astrigera L. Koch, 1878 & 2 & & & & 15 & 游猎型 \\
\hline 蒙古豹蛛 Pardosa mongolica Kulczyn'ski, 1901 & & & & & 3 & 游猎型 \\
\hline 申氏豹蛛 Pardosa schenkeli Lessert, 1904 & 8 & 53 & 21 & & 1 & 游猎型 \\
\hline
\end{tabular}


张宇, 王露雨, 向昌林, 段美春, 张志升 (2021) 不同放牧强度对赛罕乌拉草原蜘蛛多样性的影响. 生物多样性, 29, 467-476. http://www.biodiversity-science.net/CN/10.17520/biods.2020214

\begin{tabular}{|c|c|c|c|c|c|c|}
\hline \multirow{2}{*}{ 物种 Species } & \multicolumn{5}{|c|}{ 个体数量 Number of individuals } & \multirow{2}{*}{$\begin{array}{l}\text { 捕食方式 } \\
\text { Foraging type }\end{array}$} \\
\hline & $\begin{array}{l}\text { 样地 I } \\
\text { Sample } \\
\text { site I }\end{array}$ & $\begin{array}{l}\text { 样地II } \\
\text { Sample } \\
\text { site II }\end{array}$ & $\begin{array}{l}\text { 样地 III } \\
\text { Sample site } \\
\text { III }\end{array}$ & $\begin{array}{l}\text { 样地 IV } \\
\text { Sample site } \\
\text { IV }\end{array}$ & $\begin{array}{l}\text { 样地V } \\
\text { Sample } \\
\text { site V }\end{array}$ & \\
\hline
\end{tabular}

\section{米图蛛科 Miturgidae}

刺佐蛛 Zora spinimana (Sundevall, 1833)

35

游猎型

猫蛛科 Oxyopidae

猫蛛属一种 Oxyopes sp.

15

6

游猎型

逍遥蛛科 Philodromidae

$34 \quad 33$

2

2

游猎型

逍遥蛛属一种 Philodromus sp.

1

1

游猎型

白斑狼逍遥蛛 Thanatus coloradensis Keyserling, 1880

游猎型

朝鲜狼逍遥蛛 Thanatus coreanus Paik, 1979

24

63

61

3

游猎型

娇长逍遥蛛 Tibellus tenellus (L. Koch, 1876)

$151 \quad 13$

游猎型

\section{盗蛛科 Pisauridae}

针盗蛛 Pisaura ancora Paik, 1969

$4 \quad 15$

游猎型

\section{跳蛛科 Salticidae}

丽亚蛛 Asianellus festivus (C. L. Koch, 1834)

弓拱猎蛛 Evarcha arcuate (Clerck, 1757)

三带怜蛛 Talavera trivittata (Schenkel, 1963)

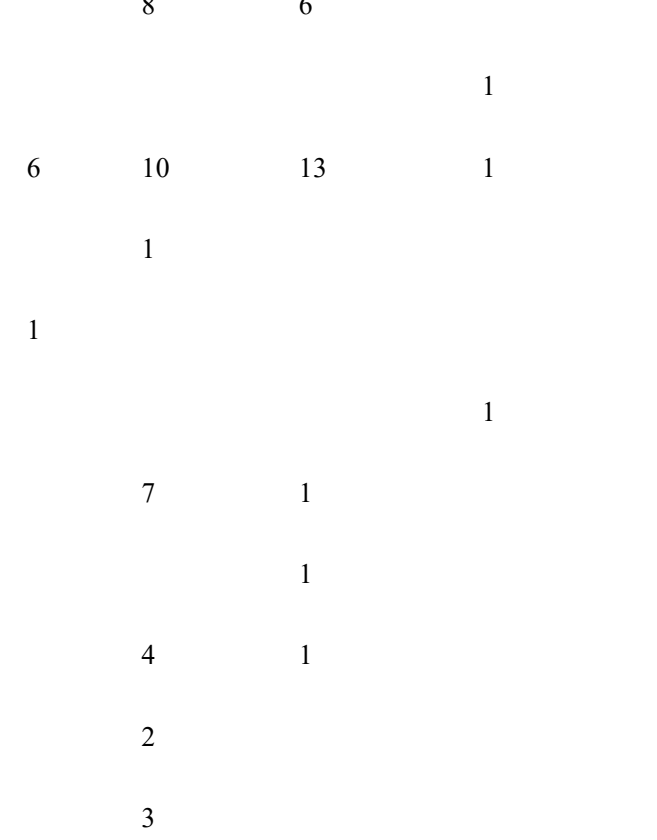

游猎型

跳蛛科一种 Salticidae sp.1

(1)

跳蛛科一种 Salticidae sp.2

跳蛛科一种 Salticidae sp.3

跳蛛科一种 Salticidae sp.4

跳蛛科一种 Salticidae sp.5

跳蛛科一种 Salticidae sp.6

跳蛛科一种 Salticidae sp.7

跳蛛科一种 Salticidae sp.8

跳蛛科一种 Salticidae sp.9

$\begin{array}{ll}6 & 10 \\ & 1 \\ & \\ & \\ & \\ & 7 \\ & \\ & \\ & \\ & \\ & \\ 3 & \end{array}$

4

游猎型

1

游猎型

游猎型

游猎型

游猎型

游猎型

游猎型

游猎型

肖蛸蛛科 Tetragnathidae 
张宇, 王露雨, 向昌林, 段美春, 张志升 (2021) 不同放牧强度对赛罕乌拉草原蜘蛛多样性的影响. 生物多样性, 29, 467-476. http://www.biodiversity-science.net/CN/10.17520/biods.2020214

\begin{tabular}{|c|c|c|c|c|c|c|}
\hline \multirow[b]{2}{*}{ 物种 Species } & \multicolumn{5}{|c|}{ 个体数量 Number of individuals } & \multirow{2}{*}{$\begin{array}{l}\text { 捕食方式 } \\
\text { Foraging type }\end{array}$} \\
\hline & $\begin{array}{l}\text { 样地 I } \\
\text { Sample } \\
\text { site I }\end{array}$ & $\begin{array}{l}\text { 样地II } \\
\text { Sample } \\
\text { site II }\end{array}$ & $\begin{array}{l}\text { 样地 III } \\
\text { Sample site } \\
\text { III }\end{array}$ & $\begin{array}{l}\text { 样地 IV } \\
\text { Sample site } \\
\text { IV }\end{array}$ & $\begin{array}{l}\text { 样地V } \\
\text { Sample } \\
\text { site V }\end{array}$ & \\
\hline 尖尾肖蛸 Tetragna caudicula (Karsch, 1879) & & & & & & 织网型 \\
\hline 羽斑肖蛸 Tetragna pinicola L. Koch, 1870 & 1 & 6 & 2 & 1 & & 织网型 \\
\hline \multicolumn{7}{|l|}{ 球蛛科 Theridiidae } \\
\hline 齿螯蛛属一种 Enoplognatha sp. & 1 & 7 & 1 & & & 织网型 \\
\hline 板隅拟肥腹蛛 Parasteatoda tabulate (Levi, 1980) & & & & 1 & & 织网型 \\
\hline 温室拟肥腹蛛 Parasteatoda tepidariorum (C.L. Koch, & 2 & & & & & 织网型 \\
\hline \multicolumn{7}{|l|}{ 1841) } \\
\hline 拟肥腹蛛属一种 Parasteatoda sp. & 1 & & & & & 织网型 \\
\hline 印痕叶球蛛 Phylloneta impressa (L. Koch, 1881) & 1 & 1 & 6 & 16 & & 织网型 \\
\hline 白斑肥腹蛛 Steatoda albomaculata (De Geer, 1778) & & & & & 5 & 织网型 \\
\hline 肥腹蛛属一种 Steatoda sp. & & & & & 7 & 织网型 \\
\hline 辽源球蛛 Theridion liaoyuanense (Zhu \& Yu, 1982) & & 8 & 4 & 1 & & 织网型 \\
\hline 球蛛属一种 Theridion sp. & & 5 & 2 & & & 织网型 \\
\hline \multicolumn{7}{|l|}{ 蟹蛛科 Thomisidae } \\
\hline 伊氏蛛属一种 Ebrechtella sp. & 2 & 17 & 5 & 4 & & 游猎型 \\
\hline 梅氏毛蟹蛛 Heriaeus melloteei Simon, 1886 & & 7 & & & & 游猎型 \\
\hline 圆花叶蛛 Synema globosum (Fabricius, 1775) & & 2 & 1 & 1 & & 游猎型 \\
\hline 满蟹蛛 Thomisus onustus Walckenaer, 1805 & 1 & & & & 1 & 游猎型 \\
\hline 岛民花蟹蛛 Xysticus insulicola Bösenberg \& Strand, & 32 & 54 & 41 & 16 & 10 & 游猎型 \\
\hline \multicolumn{7}{|l|}{1906} \\
\hline 条纹花蟹蛛 Xysticus striatipes L. Koch, 1870 & & & 2 & 2 & 3 & 游猎型 \\
\hline \multicolumn{7}{|l|}{ 隐石蛛科 Titanoecidae } \\
\hline 黄冠隐石蛛 Titanoeca flavicoma L. Koch, 1872 & 1 & & & & 3 & 游猎型 \\
\hline 合计 Total & 217 & 497 & 243 & 94 & 70 & \\
\hline
\end{tabular}

